\title{
Association of lung function, chest radiographs and clinical features in infants with cystic fibrosis
}

\author{
Margaret Rosenfeld, Philip M. Farrell, Margaret Kloster, Jonathan O. Swanson, \\ Thuy Vu, Lyndia Brumback, James D. Acton, Robert G. Castile, Andrew A. Colin, \\ Carol K. Conrad, Meeghan A. Hart, Gwendolyn S. Kerby, Peter W. Hiatt, \\ Peter J. Mogayzel, Robin C. Johnson and Stephanie D. Davis
}

Affiliations: For a list of the authors' affiliations, see the Acknowledgements.

Correspondence: M. Rosenfeld, Division of Pulmonary Medicine, Seattle Children's Hospital, 4800 Sandpoint Way NE, Seattle, WA 98105, USA. E-mail: Margaret.rosenfelddaseattlechildrens.org

ABSTRACT The optimal strategy for monitoring cystic fibrosis lung disease in infancy remains unclear. Our objective was to describe longitudinal associations between infant pulmonary function tests, chest radiograph scores and other characteristics.

Cystic fibrosis patients aged $\leqslant 24$ months were enrolled in a 10 -centre study evaluating infant pulmonary function tests four times over a year. Chest radiographs $\sim 1$ year apart were scored using the Wisconsin and Brasfield systems. Associations of infant pulmonary function tests with clinical characteristics were evaluated with mixed effects models.

The 100 participants contributed 246 acceptable flow/volume (forced expiratory volume in $0.5 \mathrm{~s}$ (FEV0.5) and forced expiratory flow at 75\% of the forced vital capacity (FEF75\%)), 303 functional residual capacity measurements and 171 chest radiographs. Both Brasfield and Wisconsin chest radiograph scores worsened significantly over the 1-year interval. Worse Wisconsin chest radiograph scores and Staphylococcus aureus were both associated with hyperinflation (significantly increased functional residual capacity), but not with diminished FEV0.5 or FEF75\%. Parent-reported cough was associated with significantly diminished forced expiratory flow at $75 \%$ but not with hyperinflation.

In this infant cohort in whom we previously reported worsening in average lung function, chest radiograph scores also worsened over a year. The significant associations detected between both Wisconsin chest radiograph score and $S$. aureus and hyperinflation, as well as between cough and diminished flows, reinforce the ability of infant pulmonary function tests and chest radiographs to detect early cystic fibrosis lung disease.

@ERSpublications

Infant pulmonary function tests and chest radiographs are able to detect early cystic fibrosis lung disease http://ow.ly/pv7pt

Received: Aug 312012 | Accepted after revision: March 182013 | First published online: May 302013

Support statement: This study was funded by the Cystic Fibrosis Foundation (ROSENF03AO and DAVIS08Y2). P.M. Farrell was supported by NIH grant Dlr34108.

Conflict of interest: Disclosures can be found alongside the online version of this article at www.erj.ersjournals.com 


\section{Introduction}

The hallmark features of cystic fibrosis (CF) lung disease include airway infection, inflammation, obstruction and structural lung damage [1-4]. These abnormalities begin in infancy [1-7], often prior to the onset of symptoms [4], and unrecognised early airway damage may progress to irreversible bronchiectasis [3]. Widespread adoption of newborn screening for CF offers the opportunity to intervene in the pre-symptomatic period in order to improve outcomes [8]. Detecting and monitoring early CF lung disease is, therefore, likely to improve long-term outcomes, but the optimal strategy for monitoring remains unclear.

Infant pulmonary function test (iPFT) measurements by the raised volume rapid thoracoabdominal compression technique (RVRTC) have been shown to be associated with lower airway infection, inflammation and bronchiectasis on computed tomography (CT) of the chest $[2,3,6,7,9]$ and to track into the preschool years $[10,11]$. While chest CT is the most sensitive means of detecting bronchiectasis in infants with CF [12], concerns remain regarding the attendant risks of anaesthesia and radiation exposure. Chest radiographs are recommended in infants as part of routine clinical care [13] and are widely employed. The association of iPFTs and chest radiograph scores in infants has not, to our knowledge, been evaluated and little is known about the association of iPFTs and other clinical characteristics, such as respiratory rate, oximetry or cough. The objective of the current study was to describe cross-sectional and longitudinal associations between iPFTs and clinical characteristics, including chest radiograph scores, in a multicentre cohort of infants with CF [1]. Portions of this work have previously been presented in abstract form [14].

\section{Methods}

Overview and study visits

This was a 10-centre, longitudinal, observational study of lung function and associated clinical characteristics in a cohort of infants with CF; details have been published previously [1]. Study visits occurred at enrolment, and at 6 and 12 months, with an additional visit within a 28-35-day window of one of these visits. At each visit, infants participated in sedated lung function testing and clinical data were collected.

\section{Participants}

Inclusion criteria included age $\leqslant 24$ months at enrolment and confirmed diagnosis of CF [15]. Exclusion criteria included: 1) acute intercurrent respiratory infection, defined as an increase in cough, wheezing, or respiratory rate in the preceding 3 weeks; 2) being currently hospitalised for a pulmonary exacerbation; and 3) oxyhaemoglobin saturation $<90 \%$ on room air. Additional exclusion criteria were related to contraindications to sedation, as previously published [1]. Institutional review board approval was obtained at each site and informed consent from the parent/guardian of each participant.

\section{Pulmonary function testing}

After sedation with $75-125 \mathrm{mg} \cdot \mathrm{kg}^{-1}$ of oral or rectal chloral hydrate (maximum $2 \mathrm{~g}$ ), plethysmography followed by RVRTC was performed with the nSpire Infant Pulmonary Lab (IPL; nSpire, Inc., Longmont, CO, USA), as previously described [1]. All PFT data were reviewed by an expert panel (S.D. Davis, R.C. Johnson and G.S. Kerby) for selection of acceptable measurements [16-18] for analyses. Based on our prior results [1], the measures analysed for this manuscript were functional residual capacity (FRC) obtained by plethysmography, forced expiratory volume at $0.5 \mathrm{~s}$ (FEV0.5) and forced expiratory flow at $75 \%$ of forced vital capacity (FEF75\%), obtained by RVRTC. z-scores for FRC were derived from the reference equations of CASTILE et al. [18] and for FEV0.5 and FEF75\% from JONES et al. [19].

\section{Clinical evaluation}

At each study visit, data were collected on quiet respiratory rate, oximetry, oropharyngeal cultures, hospitalisations, medications, length, weight and parent-reported cough $(0=$ none, $1=$ rare, $2=$ in the morning, and 3 =productive cough) [20]. At enrolment, additional data were collected on sex, race, birth history, CF genotype, family history of asthma and environmental allergies, cigarette smoke exposure, and $\mathrm{CF}$ diagnosis through newborn screening. CF genotype functional class was categorised according to the method of MCKone et al. [21] and GreEN et al. [22], as follows: minimal CF transmembrane regulator (CFTR) function, both alleles with mutations in functional class 1, 2 or 3; and residual function, one or both alleles with mutations in CFTR functional class 4 or 5.

\section{Chest radiographs and scoring}

Chest radiographs were obtained within 90 days of the enrolment and at the 12-month study visit. They were scored centrally by a reviewer (P.M. Farrell) who has $>20$ years of experience in quantitative radiographic assessment of lung disease, and who were blinded to participant identity, age and date. 
Posterior-anterior and lateral radiographs were scored concurrently by the Wisconsin [23] and Brasfield [24] systems, in batches of 50. Each session began with scoring six standard calibration films to maximise inter-session consistency. The scorer entered results onto standardised paper report forms. Scores were then entered in duplicate into an Access database. In order to evaluate intra-rater reliability, duplicates of 30 study films were inserted randomly into the set of films to be scored. The scorer was blinded to the presence of these additional films. The Wisconsin score was calculated by the additive method [23]; the possible scores range from 0 (mildest) to 100 (most severe). Possible Brasfield scores range from 25 (mildest) to 0 (most severe) [24].

\section{Statistical analysis}

Participant characteristics were summarised with standard descriptive statistics. Intra-rater reliability of chest radiograph scores was assessed with the intraclass correlation coefficient. When more than one chest radiograph score was available for a film, one score was chosen randomly for all analyses. The correlation between Brasfield and Wisconsin chest radiograph scores at enrolment was assessed using the Spearman correlation coefficient with the confidence interval determined by bootstrap methods. Because cough is a hallmark clinical characteristic of early CF lung disease [16], we evaluated the association of chest radiograph score and parent-reported cough at the nearest study visit, using generalised linear mixed effects models using a logit link with a random intercept to account for repeated measures. Based on the Wisconsin Neonatal Screening Trial we chose a Wisconsin score of 5 and a Brasfield score of 21 to define the threshold value for transition to potentially irreversible abnormalities [25].

Associations of each lung function parameter with clinical characteristics at the same visit were evaluated using mixed effects models with a random intercept to allow the use of data from all visits and account for repeated measures. Models were adjusted for age, sex and length (time-varying). The effect of baseline clinical characteristics on change from baseline in lung function z-score over the study period was assessed using mixed effects models with random subject and age effects and an interaction between age and the clinical measure. Slope estimates for change in chest radiograph scores with age were derived from a mixed effects model with random intercept. No adjustment was made for multiple comparisons in this hypothesisgenerating study. Analyses were conducted using R statistical package version 2.13 (R Foundation for Statistical Computing, Vienna, Austria).

\section{Results}

Participants and data collected

100 participants from 10 sites were enrolled between 2003 and 2006. 246 acceptable RVRTC measurements were obtained from 90 participants and 303 acceptable FRC measurements from 96 participants. 171 chest radiographs were obtained from 98 participants ( 96 from enrolment, three from the 6-month visit and 72 from the 12 -month visit). The mean $\pm \mathrm{SD}$ time between chest radiographs and the corresponding lung function tests was $-9.1 \pm 24.9$ days; median time was 0 days.

Enrolment characteristics of the study cohort are displayed in table 1 . The mean \pm SD age at enrolment was $14 \pm 6.2$ months. Most participants $(78 \%)$ had minimal CFTR function (mutations on both alleles in functional class 1, 2 or 3). 21\% were diagnosed by newborn screening. Average height and weight percentiles were slightly below the general population mean. Almost half of parents reported no cough and $11 \%$ reported cigarette smoke exposure either in utero or after birth. Because of the observed distribution of parent-reported cough, cough was dichotomised as present/absent in all subsequent analyses. Mean $\pm \mathrm{SD}$ FEF75\% Z-score at enrolment was mildly reduced $(-0.47 \pm 1.5)$ and mean FRC $\mathrm{z}$-score slightly elevated $(1.77 \pm 2.5)$. Lower FEF75\% z-scores indicate airflow limitation and higher FRC $\mathrm{z}$-scores indicate hyperinflation. Chest radiograph scores were in general in the mild range, with the mean \pm SD Brasfield score $22 \pm 1.5$ and the mean \pm SD Wisconsin score $3.5 \pm 2.7$.

\section{Chest radiographs}

Based on duplicate scoring of 30 radiographs selected at random, the intraclass correlation coefficient was 0.50 (95\% CI 0.17-0.73) for the Brasfield system and 0.56 (95\% CI 0.27-0.77) for the Wisconsin system. At enrolment, six out of 96 participants had no abnormalities detected with the Wisconsin system $($ score $=0)$ and the same six had no abnormalities detected with the Brasfield system (score $=25)$. The correlation between Wisconsin and Brasfield scores at enrolment was $\mathrm{r}=-0.75$ (95\% CI $-0.67--0.81, \mathrm{p}<0.001)$ (fig. 1). Wisconsin and Brasfield chest radiograph scores both worsened significantly with age during the 1-year study period (Wisconsin mean slope per year 0.86, 95\% CI 0.31-1.41, p=0.005; Brasfield mean slope per year $-0.71,95 \%$ CI $-1.01--0.41, \mathrm{p}<0.001$ ) (fig. 2). Among the 141 chest radiographs, $40(23 \%)$ had a Wisconsin score $\geqslant 5$ and $41(24 \%)$ had a Brasfield score $<21$, suggesting a transition to potentially irreversible lung disease [25]. 25 chest radiographs had both a Wisconsin score $\geqslant 5$ and a Brasfield score 
TABLE 1 Characteristics of the participants at enrolment

Subjects

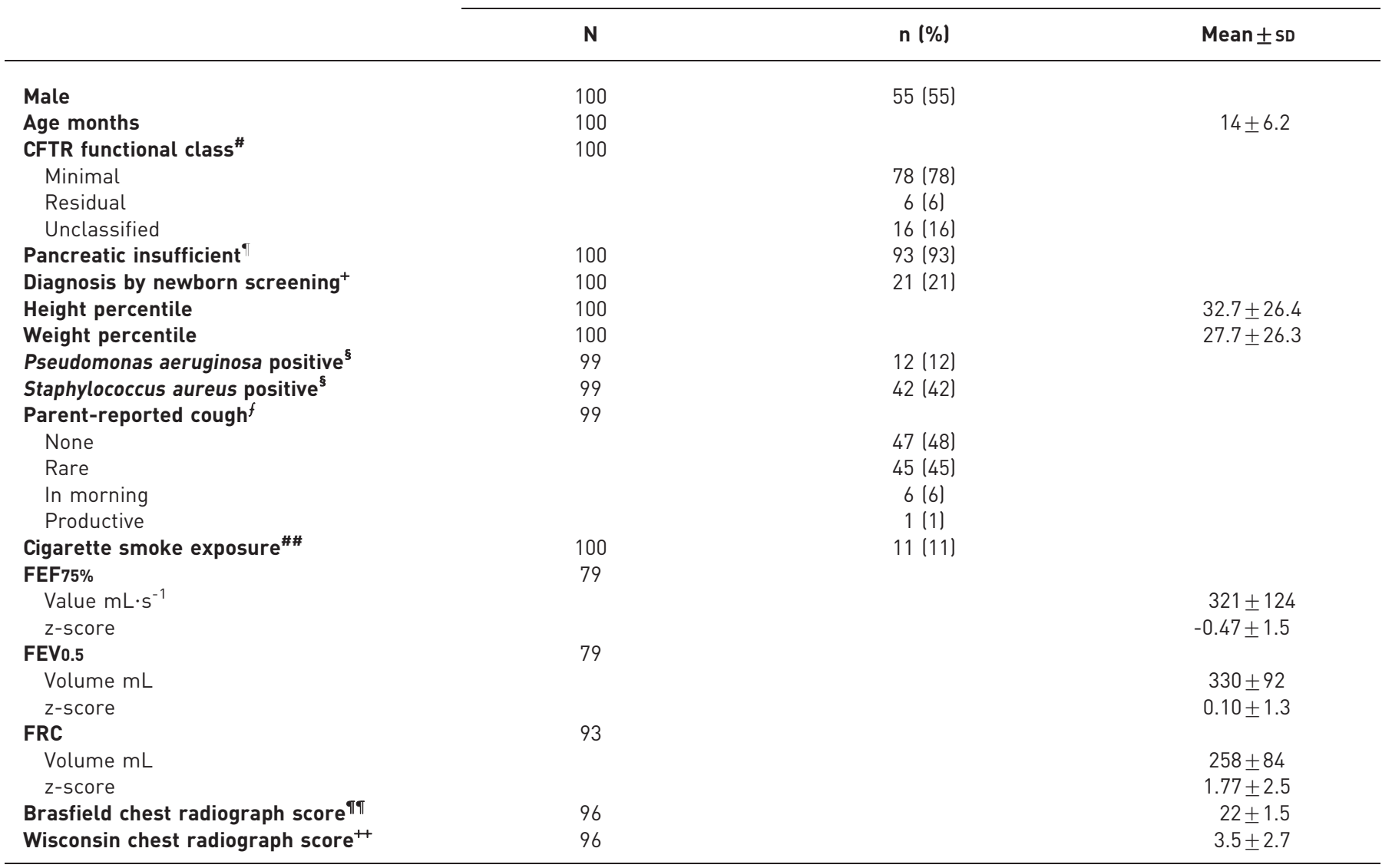

CFTR: cystic fibrosis transmembrane conductance regulator; FEF75\%: forced expiratory flow at 75\% of forced vital capacity; FEV0.5: forced expiratory volume in $0.5 \mathrm{~s}$; FRC: functional residual capacity. " : minimal, both CFTR mutations in functional class 1, 2 or 3; residual, one or both mutations in functional class 4 or $5[17,18] .{ }^{\circ}$ : reported use of pancreatic enzyme replacement therapy. ${ }^{+}$: newborn screening for cystic fibrosis was being performed at only two of the participating centres at the time of study enrolment, the University of Colorado and Children's Hospital Boston. §: based on enrolment oropharyngeal culture. ${ }^{f}$ : according to [20]. \#\# : mother smoked during pregnancy or child exposed to smoke after birth, by parent report. " ${ }^{~: ~ s c o r e s ~ c a n ~ r a n g e ~ f r o m ~} 0$ to 25 with a higher score indicating milder disease. ${ }^{++}$: scores can range from 0 to 100 with a higher score indicating more severe disease.

$<21$. There was no association between either score or presence of parent-reported cough: OR 1.62 (95\% CI 0.71-3.67) for Wisconsin score $\geqslant 5$ and OR 1.20 (95\% CI 0.53-2.73) for Brasfield score $<21$. (An OR $>1$ indicates that a worse chest radiograph score is associated with the presence of cough.)

\section{Lung function}

As previously published [1], mean (95\% confidence interval) z-scores for all acceptable lung function measures were as follows: FRC 1.92 (1.39-2.45), FEV0.5\% $0.06(-0.15-0.26)$, FEF75\% -0.52 (-0.78- -0.25). In addition, FEV0.5\% worsened significantly with increasing age (slope -0.6, 95\% CI -0.83- -0.37) and there was a trend towards worse FEF75\% and FRC with increasing age [1].

\section{Associations of lung function and clinical characteristics}

The associations of each clinical characteristic with each lung function parameter at the same study visit are displayed in table 2, from mixed effects models. There was a significant association between Wisconsin chest radiograph score and FRC: a 1-unit higher (worse) chest radiograph score was associated with a mean 4.62 (95\% CI 0.66-8.59) $\mathrm{mL}$ higher (worse) FRC. There was also a significant association between the isolation of Staphylococcus aureus from the most recent respiratory culture and FRC: a culture positive for $S$. aureus was associated with a mean $19.3 \mathrm{~mL}$ (95\% CI 5.1-33.4) higher FRC. Parent-reported cough was associated with a mean $34.6 \mathrm{~mL} \cdot \mathrm{s}^{-1}(95 \%$ CI $64.2-5.1)$ lower FEF75\%. None of these clinical characteristics was associated with both worse hyperinflation (FRC) and airway obstruction (FEV0.5 and FEF75\%). 
FIGURE 1 Correlation between Wisconsin and Brasfield chest radiograph scores at enrolment. Wisconsin scores can range from 0 (mildest) to 100 (most severe), and Brasfield scores from 25 (mildest) to 0 (most severe). Brasfield scores are jittered to allow for visibility of all data points.

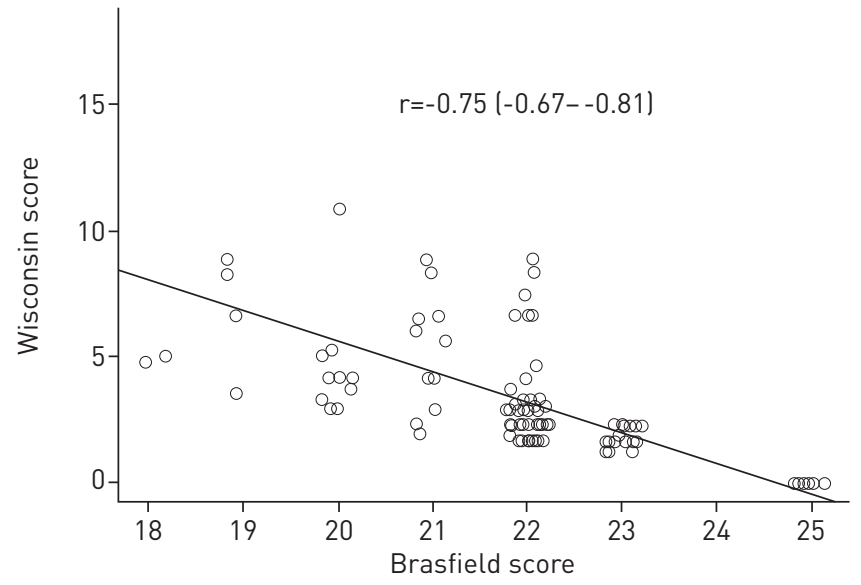

Significant associations were not detected between any lung function parameter and cigarette smoke exposure, Pseudomonas aeruginosa culture status, CF genotype functional class, weight percentile, Brasfield chest radiograph score, respiratory rate or oximetry.

The effect of baseline $P$. aeruginosa culture status, baseline weight percentile, CFTR functional class and cigarette smoke exposure on the change from baseline in lung function z-scores (FEV0.5, FEF75\% and FRC) was assessed by including an interaction between age and the clinical measure in a mixed effects model with random intercept and slope. No significant effect of any baseline characteristic on rate of change of any lung function parameter was detected.

\section{Discussion}

In this US multicentre cohort of infants with CF in whom we previously reported a worsening in average lung function [1], we now report that mean Brasfield and Wisconsin chest radiograph scores also worsen significantly over a 1-year interval. We also found that worse Wisconsin chest radiograph score and $S$. aureus respiratory infection were significantly associated with hyperinflation (higher FRC), and that parent-reported cough was significantly associated with peripheral airway obstruction (lower FEF75\%). The reason that we did not observe physiologically consistent effects of these characteristics on both hyperinflation and airway obstruction is unclear.

Chest CTs have been shown to detect bronchiectasis as early as 3 months of age [12], but are expensive and carry risks associated with radiation and anaesthesia. In school-age children and adolescents with CF, SANDERS et al. [26] recently showed that chest radiographs were important predictors of future lung dysfunction, performing as well as CT scans, and that Wisconsin and Brasfield chest radiograph scores had excellent sensitivity for detecting abnormal chest CTs [27]. The Wisconsin CF Radiograph Scoring System was specifically developed as an alternative to older scoring systems, such as the Brasfield, to be sensitive to mild CF lung disease [19], although neither system was developed to detect the very mild abnormalities now generally seen in infants and toddlers, such as those in our cohort.
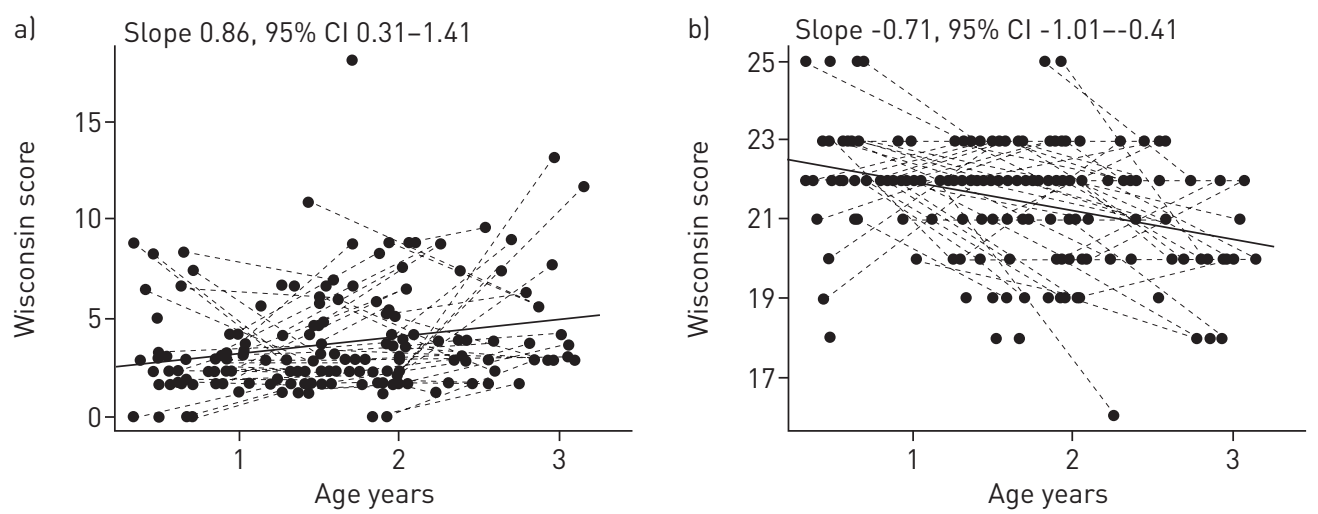

FIGURE 2 Longitudinal a) Wisconsin and b) Brasfield chest radiograph scores by age. Grey dotted lines connect measures within the same subject. Solid line represents the estimated average trend in chest radiograph score with age. 


\begin{tabular}{|c|c|c|c|c|c|}
\hline Clinical characteristics & $\begin{array}{l}\text { Observations } \\
\text { subjects }\end{array}$ & FEV $0.5 \mathrm{~mL}$ & FEF75\% $\mathrm{mL} \cdot \mathrm{s}^{-1}$ & $\begin{array}{l}\text { Observations } \\
\text { subjects }\end{array}$ & FRC $\mathrm{mL}$ \\
\hline \multicolumn{6}{|l|}{ Continuous predictors } \\
\hline Weight percentile & $246(90)$ & $0.02(-0.39-0.42)$ & $-0.23(-1.09-0.63)$ & $303(96)$ & $-0.15(-0.50-0.20)$ \\
\hline Brasfield score & 132 (87) & $-4.62(-11.33-2.09)$ & $-9.49(-24.67-5.68)$ & 154 (92) & $-5.96(-12.45-0.53)$ \\
\hline Wisconsin score & 132 (87) & $2.81(-1.85-7.48)$ & $0.93(-9.67-11.53)$ & 154 (92) & $4.62(0.66-8.59)$ \\
\hline Respiratory rate & $246(90)$ & $-0.70(-1.53-0.13)$ & $-1.21(-2.93-0.52)$ & $303(96)$ & $0.61(-0.04-1.26)$ \\
\hline Oximetry & $246(90)$ & $-0.99(-5.67-3.70)$ & $-0.17(-9.96-9.63)$ & $303(96)$ & $-0.75(-1.56-0.07)$ \\
\hline \multicolumn{6}{|l|}{ Categorical predictors } \\
\hline Cigarette smoke exposure $\#$ & $246(90)$ & $-13.0(-44.1-18.1)$ & $-27.4(-96.2-41.4)$ & $303(96)$ & $24.4(-10.3-59.0)$ \\
\hline $\begin{array}{l}\text { Pseudomonas aeruginosa } \\
\text { isolated from most recent } \\
\text { culture }\end{array}$ & $194(89)$ & $-19.2(-42.2-3.9)$ & $-37.9(-85.8-10.0)$ & $235(96)$ & $17.3(-1.3-36.0)$ \\
\hline $\begin{array}{l}\text { Staphylococcus aureus isolated } \\
\text { from most recent culture }\end{array}$ & 194 (89) & $6.9(-10.7-24.5)$ & $4.4(-32.6-41.4)$ & $235(96)$ & $19.3(5.1-33.4)$ \\
\hline Parent-reported cough & 245 (90) & $-12.6(-26.8-1.7)$ & $-34.6(-64.2--5.1)$ & $302(96)$ & $-9.4(-20.6-1.9)$ \\
\hline 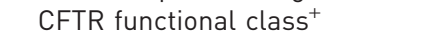 & 246 (90) & & & $303(96)$ & \\
\hline Residual function & & $32.8(-15.6-81.2)$ & $35.8(-70.4-142.0)$ & & $14.9(-34.6-64.4)$ \\
\hline Unclassified & & $4.7(-22.5-31.8)$ & $-15.9(-76.0-44.2)$ & & $2.0(-26.6-30.5)$ \\
\hline
\end{tabular}

Data are presented as $\mathrm{N}(\mathrm{n})$ or estimate $(95 \% \mathrm{CI})$. FEV0.5: forced expiratory volume in $0.5 \mathrm{~s}$; FEF75\%: forced expiratory flow at $75 \%$ of forced vital capacity; FRC: functional residual capacity; CFTR: cystic fibrosis transmembrane conductance regulator. For continuous measures, estimated mean change in infant pulmonary function test (iPFT) measure for every one unit change in clinical measure. For categorical measures, estimated mean difference in iPFT measure between categories. Mixed effects models with a random intercept were employed; all models were adjusted for length, age and sex. Significant results are presented in bold. ${ }^{\#}$ : collected at baseline visit only; ${ }^{\circ}$ : any cough versus none; ${ }^{+}$: compared with minimal CFTR function (both alleles in functional class 1, 2 or 3), residual CFTR function classified as at least one allele in functional class 4 or 5 [17, 18].

To our knowledge, ours is the first study to compare Wisconsin and Brasfield chest radiograph scores specifically in infants, to evaluate their changes over time in this age range and to compare chest radiograph scores with iPFTs. Both scoring systems were able to detect abnormalities, though not necessarily in the same participants. Our results do not suggest that one system is superior to the other in this age range. Intra-rater variability was only fair (intra-class correlation coefficient $($ ICC $)=0.5$ ) for both scoring systems in the best possible scenario (a single, highly trained scorer). In fact, intra-rater variability for CF-specific CT scoring systems is better (ICC $0.8-0.95$ in published studies) $[28,29]$. In addition, variability would be likely to be greater with less experienced or multiple scorers, reducing the utility of chest radiograph scoring. Our results suggest that, while both Brasfield and Wisconsin scores may be appropriate scoring systems in infants, with similar abilities to detect abnormalities, they may be challenging to implement in a manner that ensures accurate and reproducible results. It is of note that both scoring systems evaluate hard-copy films rather than digitised electronic images. It is possible that adapting the scoring systems to digital images could improve intra- and inter-rater variability.

Our finding of an association between lung function (FRC) and recent $S$. aureus infection complements the recent finding by PILLARISETTI et al. [2], who found that, in a cohort of newborn-screened Australian infants, FEV0.5 Z-scores declined significantly more rapidly in those infected with S. aureus. Both studies suggest a potential adverse effect of $S$. aureus on lung function in infancy. In addition, in the Wisconsin Neonatal Screening Trial, FARrell et al. [25] found an association between Wisconsin chest radiograph score and the occurrence of two respiratory cultures positive for $S$. aureus in the first 2 years of life. In contrast to PillarisetTi et al. [2], as well as our findings in preschool children with CF [11, 30], we did not detect a significant effect of $P$. aeruginosa infection on lung function. This may have been due to the small number of infants who acquired Pseudomonas in our cohort, as the observed trends in both airway obstruction (lower FEV0.5 and FEF75\%) and hyperinflation (higher FRC) were in the biologically plausible direction. Our finding of an association between cough and peripheral airway obstruction (FEF75\%) corroborates that of KOZLOWSKA et al. [10] in the London CF Collaborative infant cohort, as well as our own finding in infant and preschool CF children [11].

Strengths of our study include the relatively large size and multicentre nature of our cohort, improving external validity, as well as rigorous quality control and centralised over-reading of all iPFT data and scoring of all chest radiographs by a single experienced scorer, minimising misclassification. Limitations include the 
short observation period (1 year per participant) and lack of more invasive tests, such as CT scans or bronchoalveolar lavage. We relied on oropharyngeal cultures for respiratory microbiology, which are known to have limited diagnostic accuracy relative to lower airway cultures [31]. In addition, because the infants in our cohort were enrolled at a relatively "mature" age (mean age at enrolment of 14 months), our results may not be generalisable to newborn infants diagnosed shortly after birth by newborn screening. Lastly, given the multiple statistical tests performed, some of our results could be due to chance and should be corroborated in other cohorts.

In conclusion, our results suggest that both infant PFTs and chest radiographs may be helpful in monitoring CF lung disease in infants, but also highlight the challenges of each of these modalities. Infant lung function tests (plethysmography and RVRTC) require sedation, extensive training and expensive equipment and are not strongly associated with clinical characteristics. Similarly, while chest radiographs may play a role in detection of early structural airway damage, the expertise required to perform reliable and accurate scoring currently limits their utility. Thus, the "holy grail" of CF infant lung disease monitoring remains elusive. The lung clearance index measured by multiple-breath washout holds promise as a sensitive and noninvasive measure of early heterogeneity of ventilation [4, 32-34], but conditions for testing in infants are still undergoing validation and it may not be more sensitive than RVRTC in infants $[4,35]$. In the future, chest magnetic resonance imaging may be used to evaluate aspects of both structure and function without the radiation risks associated with CT, but is still undergoing validation [36]. In fact, monitoring of lung health in infants with CF will probably always require multiple modalities that provide complementary information on lung structure, function and infection and inflammation status. Currently, the risk-tobenefit ratio of more invasive and more accurate tools, such as CT scans, bronchoalveolar lavage and sedated iPFTs, and less invasive but less accurate tools, such as chest radiographs and oropharyngeal cultures, must be weighed on an individual basis.

\section{Acknowledgements}

The authors' affiliations are: M. Rosenfeld, Dept of Paediatrics, Division of Pulmonary Medicine, University of Washington School of Medicine and Seattle Children's Hospital, Seattle, WA, USA; P.M. Farrell, University of Wisconsin School of Medicine and Public Health, Madison, WI, USA; M. Kloster, Cystic Fibrosis Therapeutics Development Network Coordinating Center, Seattle Children's Hospital, Seattle, WA, USA; J.O. Swanson, Dept of Radiology, Seattle Children's Hospital, Seattle, WA, USA; T. Vu, Dept of Radiology, University of North Carolina School of Medicine, Chapel Hill, NC, USA; L. Brumback, Dept of Biostatistics, University of Washington, Seattle, WA, USA; J.D. Acton, Cincinnati Children's Hospital Medical Center, Cincinnati, OH, USA; R.G. Castile, Dept of Paediatrics, Center for Perinatal Research, Nationwide Children's Hospital, The Ohio State University School of Medicine and Public Health, Columbus, OH, USA; A.A. Colin, Dept of Paediatrics, University of Miami School of Medicine, Miami, FL, USA; C.K. Conrad, Dept of Paediatrics, Stanford University School of Medicine and Lucille Packard Children's Hospital, Stanford, CA, USA; M.A. Hart, Dept of Paediatrics, Case Medical Center University Hospitals of Cleveland Rainbow Babies and Children's Hospital, Cleveland, OH, USA; G.S. Kerby, Dept of Paediatrics, The Children's Hospital and University of Colorado Denver, Aurora, CO, USA; P.W. Hiatt, Dept of Paediatrics, Baylor School of Medicine, Houston, TX, USA; P.J. Mogayzel, Dept of Paediatrics, Johns Hopkins School of Medicine, Baltimore, MD, USA; R.C. Johnson, Dept of Paediatrics, Division of Paediatric Pulmonology, North Carolina Children's Hospital, University of North Carolina at Chapel Hill, Chapel Hill, NC, USA; S.D. Davis, Dept of Paediatrics, Division of Paediatric Pulmonology, North Carolina Children's Hospital, University of North Carolina at Chapel Hill, Chapel Hill, NC, and Section of Paediatric Pulmonology, Allergy and Sleep Medicine, James Whitcomb Riley Hospital for Children, Indiana University School of Medicine, Indianapolis, IN, USA.

We would like to thank S. Wilcox (University of Colorado, Denver, CO, USA) for her invaluable assistance in evaluating lung function tests and A. Laxova for assisting with the chest radiograph storing procedures. We would like to thank all the patients and families who participated in this study, as well as all the research coordinators who made the study possible.

\section{References}

1 Davis SD, Rosenfeld M, Kerby GS, et al. Multicenter evaluation of infant lung function tests as cystic fibrosis clinical trial endpoints. Am J Respir Crit Care Med 2010; 182: 1387-1397.

2 Pillarisetti N, Williamson E, Linnane B, et al. Infection, inflammation, and lung function decline in infants with cystic fibrosis. Am J Respir Crit Care Med 2011; 184: 75-81.

3 Mott LS, Park J, Murray CP, et al. Progression of early structural lung disease in young children with cystic fibrosis assessed using CT. Thorax 2012; 67: 509-516.

4 Hoo AF, Thia LP, Nguyen TT, et al. Lung function is abnormal in 3-month-old infants with cystic fibrosis diagnosed by newborn screening. Thorax 2012; 67: 874-881.

5 Linnane BM, Hall GL, Nolan G, et al. Lung function in infants with cystic fibrosis diagnosed by newborn screening. Am J Respir Crit Care Med 2008; 178: 1238-1244.

6 Peterson-Carmichael SL, Harris WT, Goel R, et al. Association of lower airway inflammation with physiologic findings in young children with cystic fibrosis. Pediatr Pulmonol 2009; 44: 503-511.

7 Nixon GM, Armstrong DS, Carzino R, et al. Early airway infection, inflammation, and lung function in cystic fibrosis. Arch Dis Child 2002; 87: 306-311.

8 Grosse SD, Rosenfeld M, Devine OJ, et al. Potential impact of newborn screening for cystic fibrosis on child survival: a systematic review and analysis. J Pediatr 2006; 149: 362-366. 
Martinez TM, Llapur CJ, Williams TH, et al. High-resolution computed tomography imaging of airway disease in infants with cystic fibrosis. Am J Respir Crit Care Med 2005; 172: 1133-1138.

10 Kozlowska WJ, Bush A, Wade A, et al. Lung function from infancy to the preschool years after clinical diagnosis of cystic fibrosis. Am J Respir Crit Care Med 2008; 178: 42-49.

11 Brumback LC, Davis SD, Kerby GS, et al. Lung function from infancy to preschool in a cohort of children with cystic fibrosis. Eur Respir J 2013; 41: 60-66.

12 Stick SM, Brennan S, Murray C, et al. Bronchiectasis in infants and preschool children diagnosed with cystic fibrosis after newborn screening. J Pediatr 2009; 155: 623-628.

13 Borowitz D, Robinson KA, Rosenfeld M, et al. Cystic Fibrosis Foundation evidence-based guidelines for management of infants with cystic fibrosis. J Pediatr 2009; 155: Suppl., S73-S93.

14 Rosenfeld M, Farrell PM, Kloster M, et al. Association of physiologic measures and clinical characteristics in a multicenter cohort of infants with CF. Pediatr Pulmonol 2011: Suppl. 34, 333.

15 Farrell PM, Rosenstein BJ, White TB, et al. Guidelines for diagnosis of cystic fibrosis in newborns through older adults: Cystic Fibrosis Foundation consensus report. J Pediatr 2008; 153: S4-S14.

16 American Thoracic Society, European Respiratory Society. ATS/ERS statement: raised volume forced expirations in infants. Am J Respir Crit Care Med 2005; 172: 1463-1471.

17 Stocks J, Godfrey S, Beardsmore C, et al. Plethysmographic measurements of lung volume and airway resistance. Eur Respir J 2001; 17: 302-312.

18 Castile R, Filbrun D, Flucke R, et al. Adult-type pulmonary function tests in infants without respiratory disease. Pediatr Pulmonol 2000; 30: 215-227.

19 Jones M, Castile R, Davis S, et al. Forced expiratory flows and volumes in infants: normative data and lung growth. Am J Respir Crit Care Med 2000; 161: 353-359.

20 Li Z, Lai HJ, Kosorok MR, et al. Longitudinal pulmonary status of cystic fibrosis children with meconium ileus. Pediatr Pulmonol 2004; 38: 277-284.

21 McKone EF, Emerson SS, Edwards KL, et al. Effect of genotype on phenotype and mortality in cystic fibrosis: a retrospective cohort study. Lancet 2003; 361: 1671-1676.

22 Green DM, McDougal KE, Blackman SM, et al. Mutations that permit residual CFTR function delay acquisition of multiple respiratory pathogens in CF patients. Respir Res 2010; 11: 140.

23 Koscik RE, Kosorok MR, Farrell PM, et al. Wisconsin cystic fibrosis chest radiograph scoring system: validation and standardization for application to longitudinal studies. Pediatr Pulmonol 2000; 29: 457-467.

24 Brasfield D, Hicks G, Soong S, et al. The chest roentgenogram in cystic fibrosis: a new scoring system. Pediatrics 1979; 63: 24-29.

25 Farrell PM, Li Z, Kosorok MR, et al. Bronchopulmonary disease in children with cystic fibrosis after early or delayed diagnosis. Am J Respir Crit Care Med 2003; 168: 1100-1108.

26 Sanders DB, Li Z, Brody AS, et al. Chest computed tomography scores of severity are associated with future lung disease progression in children with cystic fibrosis. Am J Respir Crit Care Med 2011; 184: 816-821.

27 Sanders DB, Li Z, Rock MJ, et al. The sensitivity of lung disease surrogates in detecting chest CT abnormalities in children with cystic fibrosis. Pediatr Pulmonol 2012; 47: 567-573.

28 de Jong PA, Tiddens HA. Cystic fibrosis specific computed tomography scoring. Proc Am Thorac Soc 2007; 4: $338-342$.

29 Aziz ZA, Davies JC, Alton EW, et al. Computed tomography and cystic fibrosis: promises and problems. Thorax 2007; 62: 181-186.

30 Ren CL, Rosenfeld M, Mayer OH, et al. Analysis of the associations between lung function and clinical features in preschool children with cystic fibrosis. Pediatr Pulmonol 2012; 47: 574-581.

31 Rosenfeld M, Emerson J, Accurso F, et al. Diagnostic accuracy of oropharyngeal cultures in infants and young children with cystic fibrosis. Pediatr Pulmonol 1999; 28: 321-328.

32 Belessis Y, Dixon B, Hawkins G, et al. Early cystic fibrosis lung disease detected by bronchoalveolar lavage and lung clearance index. Am J Respir Crit Care Med 2012; 185: 862-873.

33 Owens CM, Aurora P, Stanojevic S, et al. Lung clearance index and HRCT are complementary markers of lung abnormalities in young children with CF. Thorax 2011; 66: 481-488.

34 Aurora P, Gustafsson P, Bush A, et al. Multiple breath inert gas washout as a measure of ventilation distribution in children with cystic fibrosis. Thorax 2004; 59: 1068-1073.

35 Lum S, Gustafsson P, Ljungberg H, et al. Early detection of cystic fibrosis lung disease: multiple-breath washout versus raised volume tests. Thorax 2007; 62: 341-347.

36 Eichinger M, Optazaite DE, Kopp-Schneider A, et al. Morphologic and functional scoring of cystic fibrosis lung disease using MRI. Eur J Radiol 2012; 81: 1321-1329. 